\title{
Quality by Design: Concepts for ANDAs
}

\author{
Robert A. Lionberger, ${ }^{1}$ Sau Lawrence Lee, ${ }^{1}$ LaiMing Lee, ${ }^{1}$ Andre Raw, ${ }^{1}$ and Lawrence X. Yu ${ }^{1,2}$
}

Received 7 December 2007; accepted 29 February 2008; published online 9 May 2008

\begin{abstract}
Quality by design is an essential part of the modern approach to pharmaceutical quality. There is much confusion among pharmaceutical scientists in generic drug industry about the appropriate element and terminology of quality by design. This paper discusses quality by design for generic drugs and presents a summary of the key terminology. The elements of quality by design are examined and a consistent nomenclature for quality by design, critical quality attribute, critical process parameter, critical material attribute, and control strategy is proposed. Agreement on these key concepts will allow discussion of the application of these concepts to abbreviated new drug applications to progress.
\end{abstract}

KEY WORDS: control strategy; critical material attributes; critical process parameters; design space; quality by design.

\section{INTRODUCTION}

The Food and Drug Administration (FDA) (1-3) and pharmaceutical industry (4-6) are talking about quality by design, and there are many important terms that are used as part of this discussion. However, industry comments indicate that there is still much confusion in the generic industry as to the meaning of quality by design and its associated nomenclature. In this paper, we provide a consistent set of definitions to provide a clearer understanding of quality by design for abbreviated new drug applications (ANDAs).

In order to describe quality by design, we must first define what we mean by quality. In a 2004 paper, Janet Woodcock (Director for the Center for Drug Evaluation and Research) defined pharmaceutical quality as a product that is free of contamination and reproducibly delivers the therapeutic benefit promised in the label to the consumer (1). Traditionally, the relationship of product attributes to product quality has not been well understood, and thus FDA has ensured quality via tight specifications based on observed properties of exhibit or clinical trail batches and constraining sponsors to use a fixed manufacturing process. In this approach, specifications are valued not because they are related to product quality, but because they are able to

The PAR is the range of experimental observations that lead to acceptable quality. A sponsor has the option of conducting experimental observations over the entire POS; in this case the POS could be equivalent to the PAR. Alternatively a sponsor may use prior knowledge, mechanistic models and trends from the PAR to draw conclusions about sensitivity over a POS that is larger than the PAR.

Opinions expressed in this manuscript are those of the authors and do not necessarily reflect the views or policies of the FDA.

${ }^{1}$ Food and Drug Administration, Office of Generic Drugs, 7519 Standish Place, Rockville, Maryland 20855, USA.

${ }^{2}$ To whom correspondence should be addressed. (e-mail: lawrence. yu@fda.hhs.gov) detect differences batch to batch that may potentially have therapeutic consequences.

FDA's emphasis on quality by design began with the recognition that increased testing does not improve product quality (this has long been recognized in other industries). The following equation indicates where quality comes from:

Pharmaceutical Quality $=f$ (drug substance, excipients, manufacturing, packaging).

In order for quality to increase, it must be built into the product. To do this requires understanding how formulation and manufacturing process variables influence product quality; this is the function $f$ in the equation above.

\section{QUALITY BY DESIGN}

We start with the assertion that Quality by Design (QbD) is a systematic approach to pharmaceutical development that begins with predefined objectives and emphasizes product and process understanding and process control, based on sound science and quality risk management (7). It means designing and developing formulations and manufacturing processes to ensure a predefined quality. Thus, QbD requires an understanding how formulation and process variables influence product quality. Relevant documents from the International Conference on Harmonization of Technical Requirements for Registration of Pharmaceuticals for Human Use (ICH), ICH Q8 (8), Pharmaceutical Development, along with ICH Q9 (9), Quality Risk Management, and ICH Q10 (10), Pharmaceutical Quality Systems, indicate on an abstract level how quality by design acts to ensure drug product quality. Especially for ANDA sponsors, who were not actively involved in the $\mathrm{ICH}$ processes, there is a need for more concrete descriptions of quality by design.

Over the past several years, pharmaceutical scientists have provided several more specific definitions of what are the elements of quality by design $(2,4)$ and a draft of an annex 
to $\mathrm{ICH}$ Q8 has been released (7). These discussions have generally focused on the development of new drugs. Drawing on these discussions and some specific aspects of the development of generic products, a QbD development process may include (Fig. 1):

- Begin with a target product profile that describes the use, safety and efficacy of the product

- Define a target product quality profile that will be used by formulators and process engineers as a quantitative surrogate for aspects of clinical safety and efficacy during product development

- Gather relevant prior knowledge about the drug substance, potential excipients and process operations into a knowledge space. Use risk assessment to prioritize knowledge gaps for further investigation

- Design a formulation and identify the critical material (quality) attributes of the final product that must be controlled to meet the target product quality profile

- Design a manufacturing process to produce a final product having these critical material attributes.

- Identify the critical process parameters and input (raw) material attributes that must be controlled to achieve these critical material attributes of the final product. Use risk assessment to prioritize process parameters and material attributes for experimental verification. Combine prior knowledge with experiments to establish a design space or other representation of process understanding.

- Establish a control strategy for the entire process that may include input material controls, process controls and monitors, design spaces around individual or multiple unit operations, and/or final product tests. The control strategy should encompass expected changes in scale and can be guided by a risk assessment.

- Continually monitor and update the process to assure consistent quality

Design of experiments (DOE), risk assessment, and process analytical technology (PAT) are tools that may be used in the QbD process when appropriate. They are not check-box requirements.

The difference between QbD for NDA and ANDA products is most apparent at the first step of the process. For an NDA, the target product profile is under development

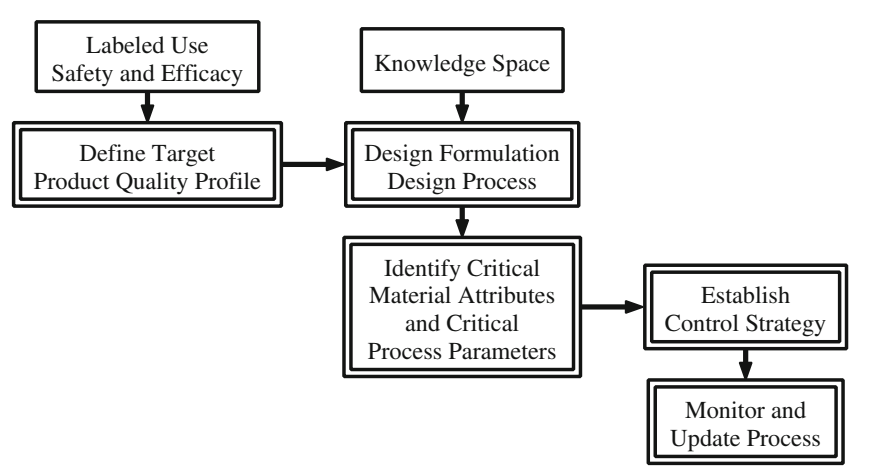

Target $\longrightarrow$ Design $\longrightarrow$ Implementation

Fig. 1. Overview of $\mathrm{QbD}$ while for the ANDA product the target product profile is well established by the labeling and clinical studies conducted to support the approval of the reference product.

\section{DESIGN GOALS}

The first aspects of QbD are an articulation of the design goals for the product.

\section{Definition of TPP}

FDA published a recent guidance defining a Target Product Profile (TPP) (11): "The TPP provides a statement of the overall intent of the drug development program, and gives information about the drug at a particular time in development. Usually, the TPP is organized according to the key sections in the drug labeling and links drug development activities to specific concepts intended for inclusion in the drug labeling." When ICH Q8 (8) says that pharmaceutical development should include "...identification of those attributes that are critical to the quality of the drug product, taking into consideration intended usage and route of administration", the consideration of the intended usage and route of administration would be through the TPP.

The TPP is a patient and labeling centered concept, it can be thought of as the "user interface" of the drug product. Thus a generic version and its reference product would be expected to have the same TPP. A generic product may use a different formulation or design to implement the TPP. The characteristics and performance tests of a drug product would depended on the particular implementation and may differ between a generic and reference product. For a new drug, changes to the TPP may require new safety or efficacy data, but changes to product characteristics or performance that result from a reformulation may not.

Many aspects of the TPP constrain or determine the actions of formulation and process development scientists. These can include the route of administration, dosage form and size, maximum and minimum doses, pharmaceutical elegance (appearance), and target patient population (pediatric formulations may require chewable tablets or a suspension). Common aspects of drug product quality are implicitly in the TPP. If the label states a tablet contains $100 \mathrm{mg}$ of active ingredient, this is a claim relating to the assay and content uniformity. It is the role of a pharmaceutical scientist to translate the qualitative TPP into what we define as the target product quality profile (TPQP) for further use in a quality by design process.

\section{Definition of TPQP}

The target product quality profile (TPQP) (12) is a quantitative surrogate for aspects of clinical safety and efficacy that can be used to design and optimize a formulation and manufacturing process. International Society of Pharmaceutical Engineers (ISPE) Product Quality Lifecycle Implementation (PQLI) calls this the Pharmaceutical Target Product Profile (4). It should include quantitative targets for impurities and stability, release profiles (dissolution) and other product specific performance requirements. Product specific examples include resuspendability for an oral suspension, adhesion for a transdermal system, and viscosity for a 
topical cream. Generic products would include bioequivalence to the RLD as part of the TPQP.

The TPQP is not a specification because it includes tests such as bioequivalence or stability that are not carried out in batch to batch release. The TPQP should only include patient relevant product performance. For example, if particle size is critical to the dissolution of a solid oral product, then the TPQP should include dissolution but not particle size. Particle size would be a critical material attribute and thus included in the process description and control strategy. The TPQP should be performance based and not mechanism based.

Examples of a TPQP can be found in the mock quality overall summary (QOS) presented on the Office of Generic Drugs website $(13,14)$ although the term TPQP was not clearly stated in the mock QOS. Another example of a TPQP is presented in the European Mock P2 (15) that was developed to facilitate a scientific and regulatory dialogue between the Industry Association European Federation of Pharmaceutical Industries Associations, and Regulatory Authorities on the presentation of enhanced product and process understanding in regulatory dossiers. The European Mock P2 uses the nomenclature Target Product Profile, but their Table I fits our definition of a TPQP. They claim that the TPQP is a definition of product intended use and a pre-definition of quality targets (with respect to clinical relevance, efficacy and safety) and thus summarizes the quality attributes of the product required to provide safety and efficacy to the patient.

\section{Definition of CQA}

The ISPE PQLI (4) defines critical quality attributes (CQAs) as physical, chemical, biological or microbiological properties or characteristics that need to be controlled (directly or indirectly) to ensure product quality. ICH Q8 (R1) defines CQAs as physical, chemical, biological or microbiological properties or characteristics that should be within an appropriate limit, range, or distribution to ensure the desired product quality (7). CQA has been used by some (16) to describe elements of the TPQP (such as dissolution) while others (17) have used CQA to describe mechanistic factors (such as particle size and hardness) that determine product performance. Thus CQA is used to describe both aspects of product performance and determinants of product performance.

It was stated that the $\mathrm{ICH}$ working definition of CQA was: "A CQA is a quality attribute (a physical, chemical, biological or microbiological property or characteristic) that must be controlled (directly or indirectly) to ensure the product meets its intended safety, efficacy, stability and performance" (18). This CQA definition implies that the intended safety, efficacy, stability and performance are not CQAs. Safety and efficacy clearly fall under the domain of the TPP. But if stability and performance are not CQA and not part of the TPP, then what are they? We are thus compelled to acknowledge that there is an intermediate category of product performance (or surrogates for quality) that we have defined as the TPQP.

As shown in Fig. 2, it seems more precise to consider the TPP, TPQP, and material attributes as separate categories. The use of CQA can be reserved for cases where there is a need to refer collectively to the targets of a $\mathrm{QbD}$ approach. CQA is generally assumed to be an attribute of the final product, but it is also possible to indicate a CQA of an intermediate or a raw material.

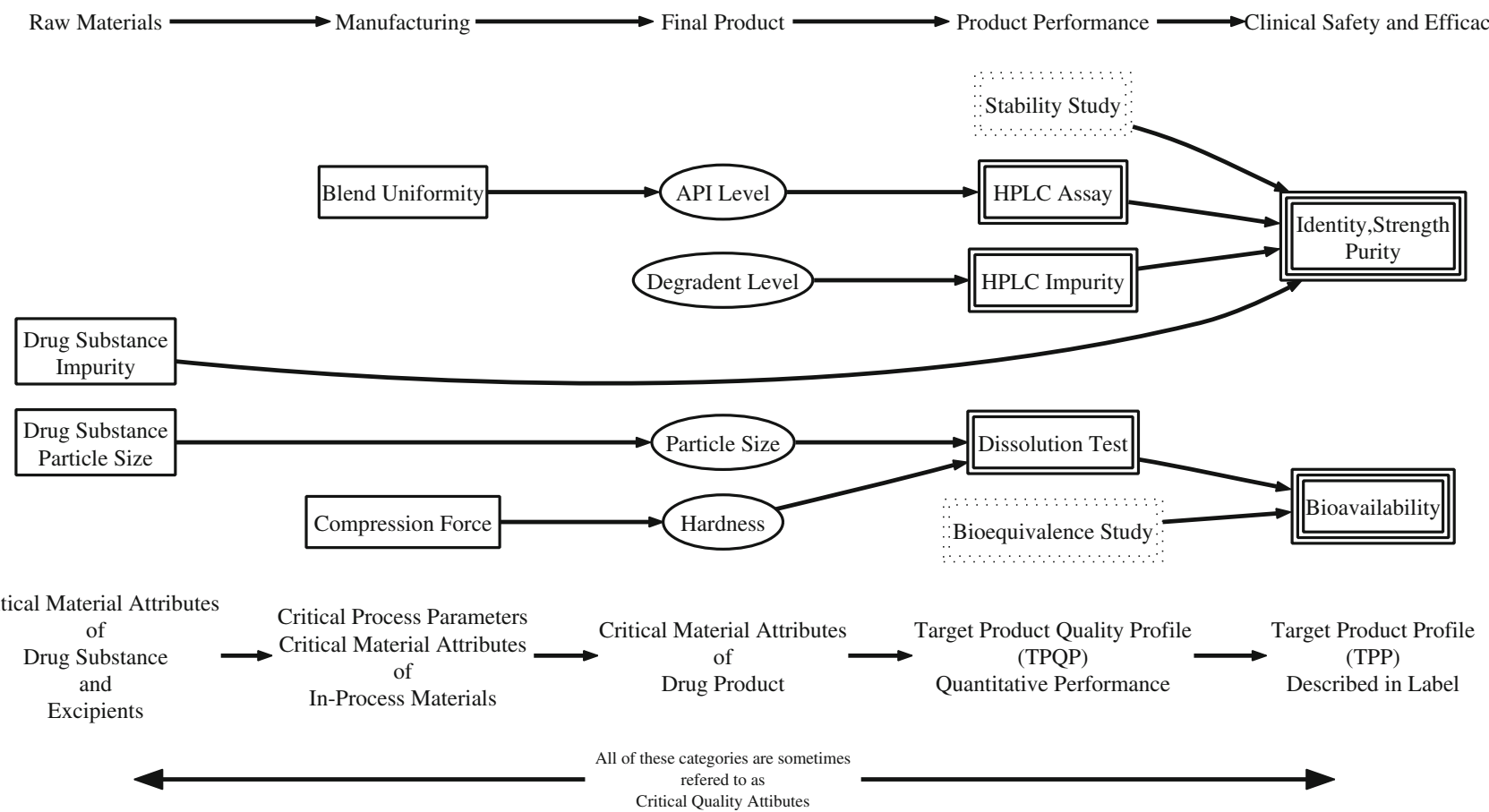

Fig. 2. An Illustration of How Under QbD the Identification of Critical Process Parameters and Critical Material Attributes is Linked to the TPQP and Finally to TPP that Represents the Clinical Safety And Efficacy 
Although many people have identified dissolution as a critical quality attribute, we consider that a set of critical material attributes (CMAs) that are independent of each other provide specific goals with which to evaluate a manufacturing process. For example a dissolution test may depend on particle size and hardness. Particle size and hardness are CMAs which can be directly linked to raw materials and manufacturing process parameters. Independent CMAs are the best way to provide a mechanistic link of the product quality to the critical process parameters in the manufacturing process. At the 2005 Drug Information Association meeting, Reed discussed dissolution in detail and indicated the greater value of have very specific CQAs (19). Others (20) have commented negatively that processing behavior of materials is usually evaluated in performance tests (flowability) rather than focusing on fundamental material properties. Differentiating between CMAs (properties) and multi-faceted performance tests is part of the movement away from quality by testing to quality by design.

The evolution of ICH Q8 is also consistent with making a distinction between CMA and performance tests. The 2004 Q8 draft (21) put CQA and performance tests into the same pile of physiochemical and biological properties:

The physicochemical and biological properties relevant to the performance or manufacturability of the drug product should be identified and discussed. These could include formulation attributes such as $\mathrm{pH}$, osmolarity, ionic strength, lipophilicity, dissolution, redispersion, reconstitution, particle size distribution, particle shape, aggregation, polymorphism, rheological properties, globule size of emulsions, biological activity or potency, and/or immunological activity. Physiological implications of formulation attributes such as pH should also be addressed.

However, the final version of Q8 (8) made clear that this section was focus on product performance:

The physicochemical and biological properties relevant to the safety, performance, or manufacturability of the drug product should be identified and discussed. This includes the physiological implications of drug substance and formulation attributes. Studies could include, for example, the development of a test for respirable fraction of an inhaled product. Similarly, information supporting the selection of dissolution $v s$. disintegration testing (or other means to ensure drug release) and the development and suitability of the chosen test could be provided in this section.

Other examples may help to show the benefit of this analysis. Consider alcohol induced dose dumping. The TPP would be the labeling statement (supported by clinical data) that the product does not dose-dump when taken with alcohol. A performance test in the TPQP would be an in vitro dissolution test in alcohol. The CMA would be the thickness of a tablet coat. Defining the CMAs on this mechanistic physical property level makes it the best link to the manufacturing process variables.

\section{CRITICAL PROCESS PARAMETERS}

\section{What is a Process Parameter?}

There is confusion about what is a process parameter. Previously, some have defined a critical process parameter (CPP) as any measurable input (input material attribute or operating parameter) or output (process state variable or output material attribute) of a process step that must be controlled to achieve the desired product quality and process consistency. In this view, every item in Fig. 3 would be a process parameter.

We propose that process parameter be understood as referring to the input operating parameters (mixing speed, flow rate) and process state variables (temperature, pressure) of a process or unit operation. Under this definition, the state of a process depends on its CPPs and the CMAs of the input materials. Monitoring and controlling output material attributes can be a better control strategy than monitoring operating parameters especially for scale up. For example, a material attribute, such as moisture content, should have the same target value in the pilot and commercial processes. An operating parameter, such as air flow rate, would be expected to change as the process scale changes.

For a given unit operation, there are four categories of parameters and attributes

- input material attributes

- output material attributes

- input operating parameters

- output process state conditions

\section{What is an Unclassified Process Parameter?}

We recognize that there are many material attributes and process parameters that are important and even essential to product quality, but it is of little value to define all parameters

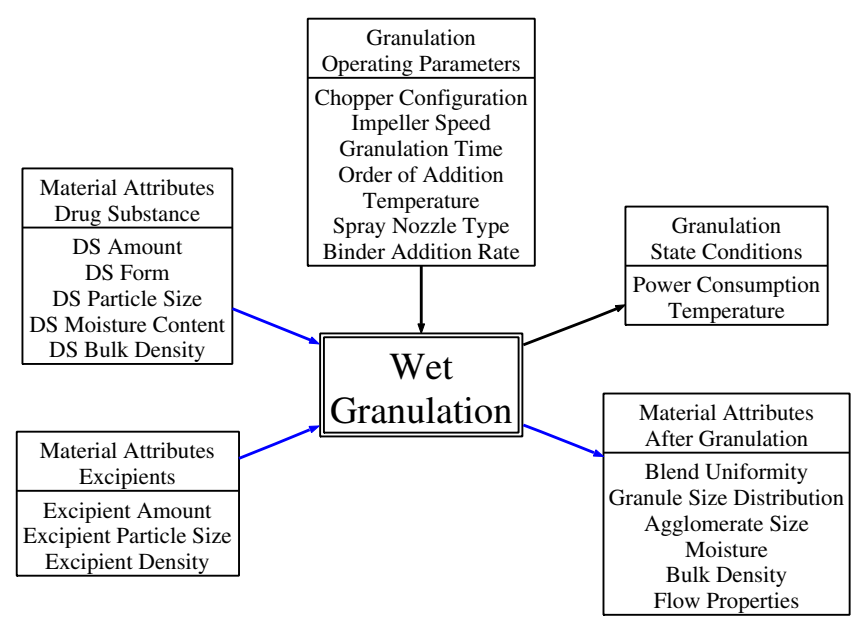

Fig. 3. An Example of Identification of Process Parameters and Material Attributes Prior to Pharmaceutical Development 
as critical. Thus we propose three categories for attributes or parameters: unclassified, critical, or non-critical. The criticality of an unclassified parameter is undetermined or unknown. Sponsors' pharmaceutical development studies can provide the additional data needed to classify an unclassified parameter as critical or non-critical. For a process or dosage form we expect wide agreement on the set of attributes or parameters that need classification. Prior experience and standard texts will guide this process. Figure 3 provides an example identification of unclassified process parameters (UPP) at the beginning of a development process.

These UPP may later be classified as critical or noncritical. For example, in the granulation process, the impeller speed should clearly be identified as an unclassified process parameter because if impeller speed were zero the process step would not be successful. However, this does not mean that impeller speed is always a critical parameter. If development studies demonstrated the granulation was not affected by realistic changes in impeller speed, it would not be identified as critical. An application that did not include the results of pharmaceutical development studies investigating the criticality of the UPP would have a large number of UPP remaining in the final submission.

\section{What is a Critical Process Parameter?}

A parameter is critical when a realistic change in that parameter can cause the product to fail to meet the TPQP. Thus, whether a parameter is critical or not depends on how large of a change one is willing to consider. A simple example is that an impeller speed of zero will always fail. Thus the first step in classifying parameters is to define the range of interest which we call the potential operating space (POS). The POS is the region between the maximum and minimum value of interest to the sponsor for each process parameter. The POS can also be considered as the extent of the sponsor's quality system with respect to these parameters. This definition is at the discretion of the application that sponsor must balance the trade-offs in its definition. The POS defines the scope of the application and the sponsor's quality system so that going outside of the POS must need an amendment or supplement to the application. Thus sponsors benefit from defining a large feasible POS. The cost of a large POS is the need for the pharmaceutical development (in the form of prior knowledge, process models or experimental data) to cover the POS and the increased chance that a parameter will be found critical in the large POS. The only constraint on the narrowness of the POS is that the POS must encompass the variability of the process parameters around their target values.

Our criteria for identifying critical and non-critical parameters are that a parameter is non-critical when there is no trend to failure within the POS and there is no evidence of interactions within the proven acceptable range (PAR)(see explanatory footnote on first page of article), which is the range of experimental observations that lead to acceptable quality. A sponsor has the option of conducting experimental observations over the entire POS; in this case the POS could be equivalent to the PAR. Alternatively a sponsor may use prior knowledge, mechanistic models and trends from the PAR to draw conclusions about sensitivity over a POS that is larger than the PAR. If the lack of interaction part of the test cannot be met, then the parameter remains a UPP. A parameter is critical when there is an observation of failure or a trend to failure predicted within the POS. If the interaction between two parameters is significant enough to predict a potential failure in the POS, then both parameters should be considered as critical.

The most definitive way to identify critical and noncritical parameters is by scientific investigations involving controlled variations of the parameters. The focus in the process development report is on the additional studies that build this knowledge. These studies can be conducted on pilot or lab scale and do not need to be conducted under current Good Manufacturing Practice. When the sensitivity of process parameters is established, this can be used to design appropriate control strategies.

However, it may not be possible (due to economic and time constraints) to conduct scientific investigations on all UPP. We believe that prior knowledge and experience with the unit operations can be used to classify some UPP. The prior knowledge can be used in a formal risk assessment process to prioritize unclassified parameters for further experimental study. This is potentially a challenging issue for FDA review, if the reviewer does not agree with the risk assessment used to classify parameters as non-critical, then all further conclusions may be in doubt because a potential critical variable was left out of the experimentation that was used to develop a design space.

Our criteria for identifying critical and non-critical process parameters are based on the sensitivity of product characteristics to changes in the process parameters. Other approaches presented in the literature link the classification as critical to the variability in a process parameter $(22,23)$. The variability of a process parameter impacts the control strategy that will be used, but we concur with ISPE PQLI that control of a variable does not render it non-critical (4). Table I summarizes the proposed classification of process parameters.

\section{Uniqueness of Critical Process Parameters}

Because of the broadness of the CPP definition it is possible for two investigators to examine the same process and come to a different set of CPP. The set of CPP is not unique, but the chosen set must be sufficient to ensure product quality.

Different sets of CPP can have several origins. One is that the definition of operating parameters depends on the engineering systems installed on a piece of process equipment. For example, one fluid bed dryer may define the product temperature as an operating parameter and have an internal control system (a thermostat) that maintains that temperature, while another fluid bed dryer may have inlet air flow rate and inlet air temperature indicated as operating parameters. The batch record for the first unit might indicate a fixed temperature, while the second unit would have a design space that indicated the combination of inlet air flow rate and inlet air temperature that would insure the appropriate product temperature.

Another source of differences in the set of CPP comes from the balance between control of operating parameters and material attributes. Morris (24) indicates that set of CPP and CMA (which he refers to as process critical control points (PCCP)) can affect the scale up process 
Table I. Classification of Process Parameters

\begin{tabular}{|c|c|c|}
\hline Parameter type & Definition & Sensitivity \\
\hline $\begin{array}{l}\text { Non-critical process } \\
\text { parameter (non-CPP) }\end{array}$ & Not critical & $\begin{array}{l}\text { - No failure in target product quality profile (TPQP) } \\
\text { observed or predicted in the potential operating } \\
\text { space (POS), and } \\
\text { - No interactions with other parameters in the proven } \\
\text { acceptable range (PAR) }\end{array}$ \\
\hline Unclassified process parameter (UPP) & Criticality unknown & $\begin{array}{l}\text { - Not established } \\
\text { - The default in the absence of pharmaceutical } \\
\text { development }\end{array}$ \\
\hline Critical process parameter (CPP) & $\begin{array}{l}\text { Critical (control needed to } \\
\text { ensure quality) }\end{array}$ & $\begin{array}{l}\text { - Failure in target product quality profile (TPQP) } \\
\text { observed or predicted in the potential operating } \\
\text { space (POS), or } \\
\text { - Interactions with other parameters in the proven } \\
\text { acceptable range (PAR) }\end{array}$ \\
\hline
\end{tabular}

- PCCPs are preserved throughout scale-up, the magnitude of the responses may not scale directly, but the variables being monitored reflect the "state" of the process

- Monitoring material properties makes scaling less equipment dependent (as opposed to only monitoring equipment properties) equipment differences (scale and type) may have an effect, however, differences in the material should reflect significant changes in the PCCPs

\section{CONTROL STRATEGY}

A control strategy may include input material controls, process controls and monitoring, design spaces around individual or multiple unit operations, and/or final product specifications used to ensure consistent quality. A control strategy is what a generic sponsor uses to ensure consistent quality as they scale up their process from the exhibit batch presented in the ANDA to commercial production.

Every process has a control strategy right now. Figure 4 shows a simplified quality assurance diagram under the current regulatory evaluation system. In this system, product quality is ensured by fixing the process to produce the active ingredient, raw material testing, performing the drug product manufacturing process as described in a fixed batch record, in-process material testing, and end product testing.

The quality of raw materials including drug substance and excipients is monitored by testing. If they meet specifications or other standards such as USP for drug substance or excipients, they can be used for manufacturing of the products. As the drug substance specification alone may not be sufficient to ensure quality, the drug substance manufacturing process is also tightly controlled. Potentially significant changes to the drug substance manufacturing process will require the drug product manufacturer to file supplements with the FDA.

The finished drug products are tested for quality by assessing if they meet specifications. In addition, manufacturers are usually expected to conduct extensive in process tests, such as blend uniformity or tablet hardness. Manufacturer are also not permitted to make changes to the operating parameters (a large number of UPPs) specified in the batch record or other process changes without filling supplements with the FDA.

This combination of fixed (and thus inflexible) manufacturing steps and extensive testing is what ensures quality under the current system. A combination of limited characterization of variability (only three pilot lots for innovator products and one pilot lot for generic products), a failure of manufactures to classify process parameters as critical or noncritical, and cautiousness on the part of regulator leads to conservative specifications. Significant industry and FDA resources are being spent debating issues related to acceptable variability, need for additional testing controls, and establishment of specification acceptance criteria. The rigidity of the current system is required because manufacturers may not understand how drug substance, excipients, and manufacturing process parameters affect the quality of their product or they do not share this information with FDA chemistry, manufacturing and controls (CMC) reviewers. Thus the FDA CMC reviewers must act conservatively.

A QbD based control strategy is shown in Fig. 5. Pharmaceutical quality is assured by understanding and controlling formulation and manufacturing variables to assure the quality of the finished product. The end product testing only confirms the quality of the product. In this example, PAT

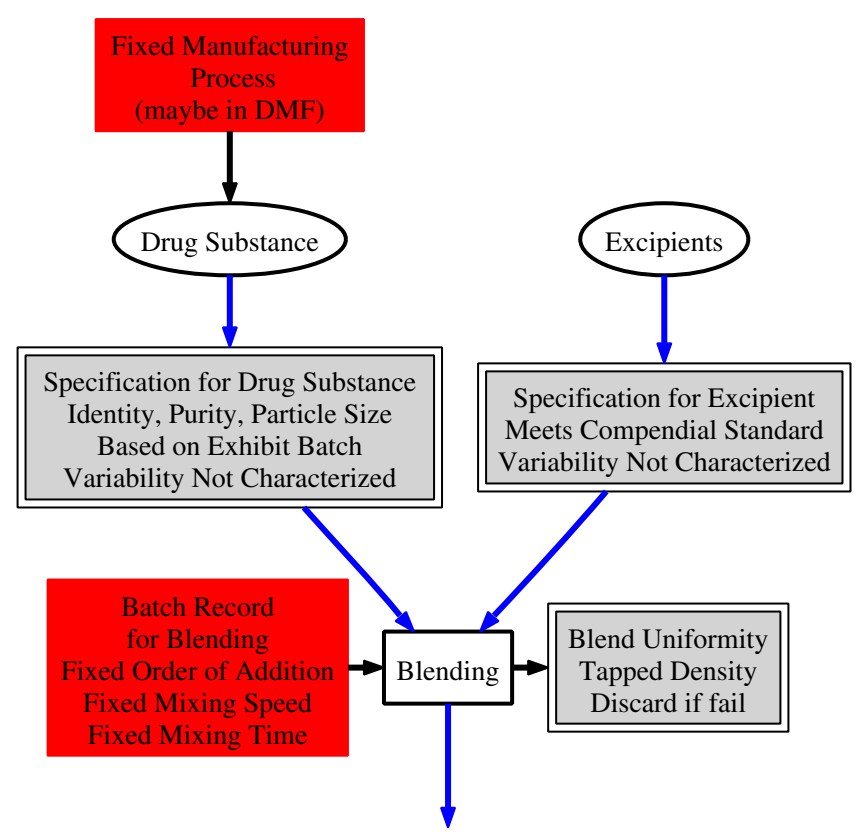

Fig. 4. An Example of Control Strategy for Pre-QbD Process 


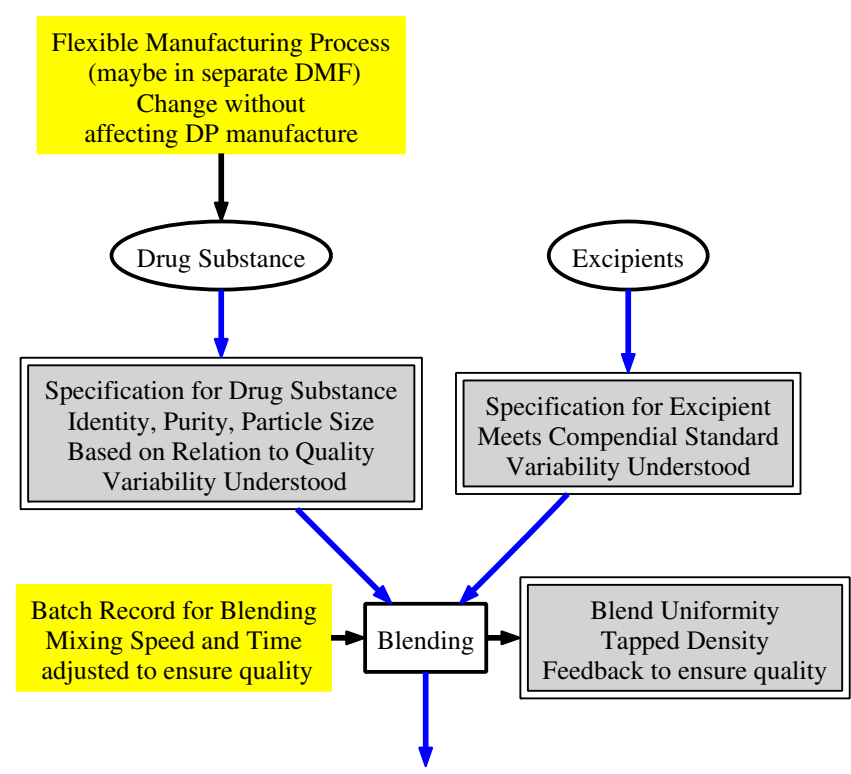

Fig. 5. An Example of Control Strategy for QbD Process

provides tools for realizing the real time release of the finished product although its use is not required under the paradigm of the Quality by Design.

\section{Implications of Process Parameter Classification}

The classification of process parameters as critical or noncritical is essential to evolve the control strategy toward the QbD based goal. Full classification of all parameters as either non-critical or critical can lead to reduced end-product testing. It is the uncertainty about the UPP that leads to extensive testing.

Without development studies, UPP may need to be constrained at fixed values or narrow ranges (used to produce acceptable exhibit batches) because they might be critical. The presence of UPP also leads to inclusion of extensive release and in-process tests into the control strategies. The goal of development studies is to move parameter from unclassified (criticality unknown) to either non-critical or critical. This classification is an important step toward a flexible manufacturing process because unclassified parameters classified as non-critical may be monitored and controlled via monovarient ranges or as part of a sponsor's quality system (see Table II). For non-critical parameters it may be possible to designate a normal operation range (NOR) up to (or beyond) the proven acceptable range (PAR) depending on trends and prior knowledge. The superposition of NOR for non-critical parameters would be considered as part of the design space.

The ranges of critical parameters must be constrained to a multidimensional design space or fixed at values of all parameters known to be acceptable. Univariate PAR can be used for critical parameters only when there is evidence that there are no significant interactions between the CPP. However the establishment of this knowledge about CPPs may render them lower risk than UPP. A control strategy appropriate to the known CPP may also have less need for release testing than one for a process with many UPPs.

\section{Design Space}

In the presence of interacting critical process parameters a design space is one approach to ensure product quality although it is not a check-box requirement. The current definition of design space is "The multidimensional combination and interaction of input variables (e.g., material attributes) and process parameters that have been demonstrated to provide assurance of quality." (8) This definition evolved from early ICH Q8 drafts where design space was defined as "the established range of process parameters that has been demonstrated to provide assurance of quality" (21). The change emphasizes the multidimensional interaction of input variables and closely binds the establishment of a design space to a conduct of a DOE that includes interactions among the input variables. A design space may be constructed for a single unit operation, multiple unit operations, or for the entire process.

Submission of a design space to FDA is a pathway obtaining the ability to operate within that design space without further regulatory approval. A design space is a way to represent the process understanding that has been established. The benefits of having a design space are clear; one challenge to the effective use of a design space is the cost of establishing it.

In a typical design space approach a sponsor identifies the unclassified parameters and then does a DOE on some of the unclassified parameters with the other unclassified parameters fixed. Thus the end is a regulatory situation where there is some space for the selected parameters but no flexibility for the other parameters. This operating parameter based design space is limited to the equipment used to develop the design space. It might change on scale up or equipment changes.

In the development of a design space, the key issue to efficiency is demonstrating or establishing that the unclassified parameters left out of the DOE are truly non-critical process parameters and are thus by our definition non-

Table II. Impact of Classification of Process Parameters on Control Strategy

\begin{tabular}{ll}
\hline \multicolumn{1}{c}{ Parameter type } & Potential control strategies \\
\hline Non-critical process parameter (non-CPP) & - Univarient range in batch record (may extend beyond existing data) \\
& - Under control of sponsors quality system \\
Unclassified process parameter (UPP) & Extensive release testing because of uncertainty \\
& - Fix at exhibit batch value or narrow range (to be tested in validation) to \\
ensure no interactions & - Reduced release testing when all critical process parameters are identified, \\
Critical process parameter (CPP) & monitored and controlled \\
& - Proven acceptable range if no evidence of multivariate interactions \\
& - Design space to allow multivariate changes \\
& - Feedback control based on measurement of material attributes
\end{tabular}


interacting. Before attempting to establish a design space, effort should be invested to reduce the number of unclassified process parameters. This may involve a screening DOE to rule out significant interactions between process parameters. When they are non-interacting, univariate ranges for noncritical parameters are appropriate and can be added to the design space presentation without additional studies.

It is best to exploit the non-uniqueness of CPPs to define the design space in terms of scale independent (dimensionless) parameters and material attributes. Understanding the design space in terms of material attributes allows scale up and equipment changes to be linked to previous experiments. The scalability of the design space can be evaluated in the transfer from lab to exhibit batch manufacturing.

\section{Feedback Control and PAT}

Application of PAT (25) may be part of a control strategy. ICH Q8(R) (7) identifies one use of PAT as ensuring that the process remains within an established design space. In a passive process, PAT tools provide continuous monitoring of CPP to demonstrate that a process is maintained in the design space. In process testing of CMA can also be conducted online or inline with PAT tools. Both of these applications of PAT are more efficient ways to detect failures. In a more robust process, PAT can enable active control of CPP, and if there is variation in the environment or input materials the operating parameters can be adjusted to keep the CMA under control to ensure quality.

A PAT system that combines continuous monitoring of CMA (instead of CPP) can potentially be combined with feedback control of process parameters to provide an alternative to design space based control strategies. A problem with design space is that it can limit flexibility. A design space is usually a specified space of process parameters that has been demonstrated to provide acceptable quality. There may be sets of process parameters that lead to acceptable quality but were not explored in the establishment of the design space. Thus, pursuit of a design space can be movement in the opposite direction from a flexible and robust manufacturing process. Direct assessment of product quality via PAT may support more flexibility and robustness than is represented by the design space. When CMA can be actively monitored and feedback control applied to the CPP, then variation in the environment or input materials can be counteracted by new values of the CPP (even values outside of a design space that represents prior experience) to keep the CMA within desired limits. When direct assessment of product quality by PAT is established, it may be more valuable to invest pharmaceutical development resources toward an active control system than toward documentation of a design space.

\section{CONCLUSIONS}

Quality by design is an essential part of the modern approach to pharmaceutical quality. This paper clarifies the use of $\mathrm{QbD}$ for ANDAs including:

- Emphasis on the importance of the Target Product Quality Profile in articulating a quantitative performance target for QbD.
- Identification of critical material attributes that provide a mechanistic link of the product quality to the manufacturing process.

- Clarification that critical process parameters are operating parameters and should be combined with critical material attributes to describe the relation between unit operation inputs and outputs.

- A definition of non-critical, unclassified, and critical that provides a way to classify process parameters and in-process material attributes

- The role of the control strategy as the mechanism for incremental implementation of $\mathrm{QbD}$ elements into practice

- An efficient path to a design space through the identification of non-interacting process variables and their exclusion from formal experimental designs.

\section{REFERENCES}

1. J. Woodcock. The concept of pharmaceutical quality. Am. Pharm. Rev. Nov/Dec 2004:pp. 1-3.

2. M. N. Nasr. Implementation of quality by design (ObD): status, challenges, and next steps. FDA Advisory Committee for Pharmaceutical Science. Available at: http://www.fda.gov/ohrms/ dockets/ac/06/slides/2006-4241s1_6.ppt (accessed 11/21/2007).

3. L. X. Yu. Implementation of quality-by-design: OGD initiatives. FDA Advisory Committee for Pharmaceutical Science. Available at: http://www.fda.gov/ohrms/dockets/ac/06/slides/2006-4241s1_ 8.ppt (accessed 11/21/2007)

4. ISPE PQLI. Draft PQLI summary update report. http://www.ispe. org/cs/pqli_product_quality_lifeycle_implementation_/draft_pqli_ summary_update_report (accessed 11/21/2007).

5. W. P. Ganzer, J. A. Materna, M. B. Mitchell, and L. K. Wall. Current thoughts on critical process parameters and API synthesis. Pharm. Technology. 46-66 (2005), July.

6. M. Glodek, S. Liebowitz, R. McCarthy, et al. Process robustness: A PQRI white paper. Pharm. Eng. 1-11 (2006), Nov/Dec.

7. ICH. Draft consensus guideline: pharmaceutical development annex to Q8. Available at:, http://www.ich.org/LOB/media/ MEDIA4349.pdf (accessed 11/21/2007).

8. FDA CDER. Guidance for industry. Pharmaceutical development. May 2006.

9. FDA CDER. Guidance for industry. Quality risk management. June 2006

10. FDA CDER. Draft guidance for industry. Pharmaceutical quality system. July 2007.

11. FDA CDER. Draft guidance for industry and review staff. Target product profile-a strategic development process tool. Mar., 2007.

12. L. X. Yu, A. Raw R. Lionberger, et al. U.S. FDA Question-based review for generic drugs: a new pharmaceutical quality assessment system. J. of Generic Med. 4:239-248 (2007).

13. FDA Office of Generic Drugs. Model quality overall summary for an extended release capsule. Available at http://www.fda.gov/ cder/OGD/QbR/OGD_Model_QOS_ER_Capsule.pdf. (accessed $11 / 21 / 2007)$

14. FDA Office of Generic Drugs. Model quality overall summary for an immediate release tablet. http://www.fda.gov/cder/OGD/ QbR/OGD_Model_QOS_IR_Tablet.pdf (accessed 11/21/2007).

15. C. Potter, R. Beerbohm A. Coups, et al. A guide to EFPIA's Mock P.2 document. Pharm. Technology Europe. 18:39-44 (2006).

16. R. Nosal. PQLI-criticality. ISPE PQLI Berlin Conference. Sept. 2007.

17. C. Tong, S. S. D-Souza, J. E. Parker, and T. Mirza. Commentary on AAPS workshop dissolution testing for the twenty-first century: Linking critical quality attributes and critical process parameters to clinically relevant dissolution. Pharm. Res. 24:1603-1607 (2007). 
18. J. Berridge. ICH Q8 \& Q9 (+Q10) defined and undefined: gaps and opportunities. ISPE PQLI Washington Conference. June 2007.

19. R. A. Reed. A quality by design approach to dissolution based on the biopharmaceutical classification system. DIA Annual Meeting, June 2005.

20. A. J. Hlinak, K. Kuriyan, K. R. Morris, G. W. Reklaitis, and P. K. Basu. Understanding critical material properties for solid dosage form design. J. Pharm. Innovation. 1:12-17 (2006).

21. FDA CDER. Draft guidance for industry. Q8 pharmaceutical development, version 4.3 (draft), Nov. 2004.

22. T. Parks. A science and risk-based approach for establishing critical process parameters and critical intermediate quality attributes. Available at: http://www.pharmachemicalireland.
ie/Sectors/IPCMF/IPCMFDoclib4.nsf/wvICCNLP/2E3576 E6D5211C69802570BC004BA9F8/\$File/12+-+CPP+Poster.ppt. (accessed 11/21/2007).

23. R. Nosal. Industry perspective of risk-based CMC assessment under QbD. AAPS Annual Meeting, Oct. 2006.

24. K. R. Morris. Risked-based development and CMC question-based review: Asking the right questions for process understanding, control and filing. FDA Advisory Committee for Pharmaceutical Science. Available at: http://www.fda.gov/ohrms/dockets/ac/04/ slides/2004-4052S1_12_Morris.ppt (accessed 11/21/2007).

25. FDA CDER. Guidance for industry: PAT-a framework for innovative pharmaceutical development, manufacturing, and quality assurance. Sept. 2004. 